\title{
Recomendaciones para el cuidado y la atención de los recién nacidos prematuros en tiempos de la pandemia de COVID-19: Revisión integrada de la literatura
}

\author{
Adriana Matos Ferraz Florindo, ${ }^{1}$ (D) Giovanna Campos Gavasso, ${ }^{1}$ (iD Jaqueline Pereira Lopes, ${ }^{1}$ \\ D Júlia de Oliveira Gomes Vieira, ${ }^{1}$ (D) Rafael de Melo, ${ }^{1}$ (D) Gustavo Gonçalves dos Santos, ${ }^{2}$ \\ (iD) Zaide Silva Frazão. ${ }^{3}$
}

\begin{abstract}
RESUMEN
Objetivo: Describir las recomendaciones para la asistencia y el cuidado de los recién nacidos prematuros en el escenario de la pandemia COVID-19.

Métodos: Revisión de literatura integrada, realizada en 2020, en bases de datos en la Biblioteca Virtual de Salud extrayendo producciones cientificas de las bases de datos: MEDLINE, SCIELO, PUBMED y LILACS, con una muestra final de 14 publicaciones. Resultados: Entre las recomendaciones destacan: en cuanto al parto, elegir el mejor momento, ruta y lugar, priorizando el uso de una sala de presión negativa. Después del parto, pinzamiento precoz del cordón umbilical, los recién nacidos deben ser limpiados y secados lo antes posible, y aislados y se les debe hacer la prueba para detección de SARS-CoV-2 tempranamente. Después de los cuidados iniciales, se recomienda el uso de una mascarilla y la higiene de las manos antes de cada comida u otro contacto cercano con el recién nacido. Idealmente, el neonato sospechoso o contaminado debe permanecer aislado y constantemente monitoreado, con un periodo de cuarentena de al menos 14 días.

Conclusiones: El conocimiento de las enfermeras y otros profesionales de la salud acerca de las medidas de prevención y control de la infección del recién nacido por la COVID-19 es de fundamental importancia para la prevención, el control y la reducción del impacto de la pandemia en la población neonatal.
\end{abstract}

Palabras clave: Infecciones por Coronavirus, COVID-19, Cuidados de enfermería, Enfermería neonatal, Infecciones de nacimiento prematuro

\section{Recommendations for care and attention of premature newborn in times of COVID-19 pandemic: Integrated Literature Review \\ SUMMARY}

Objective: To describe recommendations for the care and handling of preterm infants in the COVID-19 pandemic scenario. Methods: Integrated Literature Review (ILR) conducted in 2020 in databases in the Virtual Health Library (VHL) by extracting scientific productions from the databases: MEDLINE, SCIELO, PUBMED and LILACS, with a final sample of 14 publications. Results: Among the recommendations found in the studies analyzed, regarding childbirth, it is recommended to choose the best time, route and place, prioritizing the use of a negative pressure room. After delivery, newborns should have the umbilical cord clamped, be cleaned and dried as soon as possible, and isolated and tested early. After initial care, the use of a mask and hand hygiene is recommended before each meal or other close contact with the newborn. Ideally, suspected or contaminated RN should be kept in isolation and constantly monitored, with a quarantine period of at least 14 days.

Conclusions: The knowledge of nurses and other health care professionals about prevention and control measures for newborn infection with COVID-19 is critical to the prevention, control, and reduction of the impact of the pandemic on the neonatal population.

Keywords: Coronavirus infections, COVID-19, Nursing Care, Neonatal Nursing and Premature Birth Infections.

\begin{abstract}
'Académicos en el curso de Pregrado en Bachiller en Enfermería. Centro Universitário das Faculdades Metropolitanas Unidas - FMU. São Paulo, SP - Brasil. ${ }^{2}$ Enfermero Obstetra y Profesor. Profesor Preceptor del Departamento de Enfermería en el curso de Enfermería de Pregrado en Centro Universitário das Faculdades Metropolitanas Unidas - FMU. Estudiante del Programa de Maestría en Salud Colectiva en Universidade Estadual Júlio de Mesquita Filho - FMB/UNESP. São Paulo, SP - Brasil. E-mail: gustavo.nahara@gmail.com. ${ }^{3}$ Master en Ciencias de la Salud.
\end{abstract}

Profesora del Departamento de Enfermería en el curso de Enfermería de Pregrado en Centro Universitário das Faculdades Metropolitanas Unidas FMU. São Paulo, SP - Brasil. E-mail: zaydy@uol.com.br.

Forma de citar este artículo: Matos A, Campos G, Pereira J, de Oliveira J, de Melo R, Goncalvez G, et al. Recomendaciones para el cuidado y la atención de los recién nacidos prematuros en tiempos de pandemia de COVID.19: revisión integrada de la literatura. Rev Obstet Ginecol Venez. 2021; 81(3):262-272. https://doi.org/10.51288/00810310 


\section{INTRODUCCIÓN}

En diciembre de 2019, un nuevo coronavirus de origen hasta ahora desconocido se propagó en la provincia de Hubei en China, causando el Síndrome Respiratorio Agudo Severo por Coronavirus 2 (SARS-CoV-2) y luego se extendió por todo el mundo (1). Este síndrome se conoció en la literatura científica como COVID-19 (coronavirus disease) y se manifiesta por un espectro clínico que va desde infecciones asintomáticas hasta condiciones graves. Según el Ministerio de Salud (MS) de Brasil, alrededor del $80 \%$ de los pacientes con la COVID-19 pueden ser asintomáticos o presentar síntomas leves, aproximadamente el $20 \%$ de los casos necesitan atención hospitalaria debido a la dificultad respiratoria, de los cuales el $5 \%$ necesitan tratamiento en una Unidad de Cuidados Intensivos (UCI) con oxigenoterapia y ventilación mecánica (2).

La vigilancia epidemiológica y las medidas de tratamiento de la COVID-19 se están construyendo a medida que la Organización Mundial de la Salud (OMS) consolida la información recibida de los países y se publican nuevas pruebas técnicas y científicas (3). En el Brasil, el escenario es incierto, ya que las estimaciones válidas del número de casos y muertes por la COVID-19 entran en conflicto con la ausencia de datos fiables y la aplicación de medidas eficaces y normalizadas a todos los niveles de gobierno $(3,4)$.

Además, la situación de extrema vulnerabilidad y desigualdad social de la población brasileña, así como las personas que viven en condiciones precarias de vivienda y saneamiento, sin acceso adecuado al agua tratada, en situaciones inevitables de aglomeración, las altas tasas de desempleo y los profundos recortes en las políticas sociales agravan el panorama de la COVID-19 en el país (4) y en muchos países de Latinoamérica.

Dada la elevada tasa de transmisión de la COVID-19, asociada a la ausencia de inmunidad previa en la población y a la ausencia de vacuna, el crecimiento del número de casos es exponencial y se necesitan medidas para detener su transmisión. Por consiguiente, es importante que todos se mantengan informados y cumplan las intervenciones no farmacológicas indicadas, que incluyen medidas de alcance individual, ambiental y comunitario, como el lavado de manos, la etiqueta de respiración, el distanciamiento físico y social, la ventilación y la exposición al sol de los ambientes, la limpieza de objetos y superficies, y la restricción o prohibición del funcionamiento de escuelas, universidades, lugares de reunión de la comunidad, el transporte público, además de otros lugares donde hay aglomeración de personas. La distancia física recomendada es de un metro o más y el uso de una máscara que cubra boca y nariz es una medida de gran importancia, especialmente para grupos de riesgo como los que padecen enfermedades crónicas como la diabetes y la hipertensión, el asma, la enfermedad pulmonar obstructiva crónica, los fumadores, los mayores de 60 años, las embarazadas, las puérperas y los niños menores de 5 años (5).

El escenario de esta pandemia ha fomentado nuevas investigaciones y planteado interrogantes sobre la atención y asistencia hospitalaria, ya que la transmisión nosocomial y el uso de procedimientos que generan aerosoles producen altas tasas de infección (6). Además de estas cuestiones, otro punto importante que debe examinarse es la gestión de la atención durante el embarazo, el parto y el recién nacido (RN), particularmente la atención en prematuros, que es un grupo especialmente vulnerable debido a su inmadurez inmunológica.

Aunque todavía hay pocos estudios, no se ha demostrado que la transmisión vertical del virus por vía transplacentaria se produzca durante el parto o la lactancia. En la mayoría de los casos, la población pediátrica presenta síntomas leves o son asintomáticos, sin embargo, los recién nacidos están más expuestos a infecciones de mayor gravedad $(7,8)$. 


\section{RECOMENDACIONES PARA EL CUIDADO Y LA ATENCIÓN DE LOS RECIÉN NACIDOS PREMATUROS EN TIEMPOS DE LA PANDEMIA DE COVID-19: REVISIÓN INTEGRADA DE LA LITERATURA}

Sobre la base de los datos científicos de China, Italia y Estados Unidos de América, los recién nacidos parecen estar significativamente menos afectados por la COVID-19 (9-11). En el Brasil, entidades nacionales como la Sociedad Brasileña de Pediatría y el Programa de Reanimación Neonatal han producido un número importante de documentos sobre este tema (12-15). Aún no se ha establecido si la COVID-19 puede presentar transmisión transplacentaria o vertical. En este momento, hay pocos casos neonatales positivos confirmados de COVID-19 que hayan sido reportados en revistas científicas, los datos señalan la ausencia de síntomas o síntomas muy leves a moderados, sin que se haya reportado ningún caso fatal en infantes en $<28$ días (9-11).

A través de este estudio es posible observar la relevancia y la importancia de los Enfermeros en el contexto de la atención y la asistencia prematura de $\mathrm{RN}$ en tiempos de la pandemia por la COVID-19. El objetivo es describir, a través de la literatura científica, las recomendaciones para la asistencia y el cuidado de los recién nacidos prematuros en ese escenario.

\section{MÉTODOS}

Se trata de un estudio de revisión de la literatura integrada (RIL) (16). Se definió la siguiente pregunta orientadora como un problema de investigación: ¿Cuáles son las recomendaciones para la asistencia y la atención del neonato prematuro en la pandemia de COVID-19?

Los criterios para la selección de artículos que se incluyeron en la revisión fueron: artículos publicados en portugués o inglés, artículos completos sobre el tema investigado, publicados e indexados en las bases de datos referidas y publicados en los últimos diez años. Se excluyeron: artículos publicados en español, artículos que no se refieran al problema y al objetivo del estudio, artículos duplicados en las bases de datos referidas y fuera del plazo de publicación.

Se realizó una búsqueda en la Biblioteca Virtual de Salud (BVS) extrayendo producciones científicas de las bases de datos: National Library of Medicine (MEDLINE), National Library of Medicine (PUBMED), Literatura Latino-Americana e do Caribe em Ciências da Saúde (LILACS) y la biblioteca virtual Scientific Electronic Library Online (SciELO). La búsqueda se realizó en el periodo de agosto a octubre de 2020, utilizando los siguientes Descriptores de Salud (DeCS) y sus combinaciones en portugués e inglés: Infecciones por Coronavirus, COVID-19, Cuidados de enfermería, Enfermería neonatal y Nacidos prematuros (tabla 1).

Tabla 1. Etapas de búsqueda y recolección de datos de la revisión de literatura integrada

\begin{tabular}{|c|c|c|c|c|}
\hline Base de dados & Estrategia de búsqueda & $\begin{array}{c}\text { Artículos } \\
\text { localizados }\end{array}$ & $\begin{array}{c}\text { Seleccionados } \\
\text { para lectura }\end{array}$ & $\begin{array}{c}\text { Incluidos } \\
\text { en el estudio }\end{array}$ \\
\hline \multirow{3}{*}{ MEDLINE } & "COVID-19" & \multirow{3}{*}{22} & \multirow{3}{*}{16} & \multirow{3}{*}{2} \\
\hline & "Cuidados de Enfermería" & & & \\
\hline & "Recién-nacidos" & & & \\
\hline SCIELO & $\begin{array}{c}\text { "COVID-19" } \\
\text { "Enfermería neonatal" }\end{array}$ & 2 & 2 & 1 \\
\hline PUBMED & $\begin{array}{c}\text { "COVID-19" } \\
\text { "Enfermería neonatal" }\end{array}$ & 11 & 11 & 10 \\
\hline LILACS & $\begin{array}{c}\text { "COVID-19" } \\
\text { "Enfermería neonatal" }\end{array}$ & 2 & 2 & 1 \\
\hline
\end{tabular}


En la presentación de los datos obtenidos se hizo una síntesis que contenía: año, autor, título y objetivo, se sometió al análisis cualitativo de los datos recogidos (tabla 2). En la búsqueda de la base de datos se aplicaron los filtros: "Texto completo", "Idioma inglés y portugués" y "últimos 10 años".
La presentación de los datos obtenidos se realizó de manera descriptiva, lo que permitió evaluar la aplicabilidad de la RIL, proporcionando una forma organizada de examinar las pruebas sobre la atención y la asistencia a los recién nacidos prematuros en el contexto de la pandemia de COVID-19.

Tabla 2. Síntesis de los resultados de la revisión de literatura integrativa según: año, autor, título y objetivo

\begin{tabular}{|c|c|c|c|}
\hline Año & Autor(es) & Título & Objetivo(s) \\
\hline 2020 & $\begin{array}{l}\text { Garcia } \mathrm{F}, \\
\text { et al. }(17)\end{array}$ & $\begin{array}{l}\text { Boas práticas no cuidado ao recém- } \\
\text { nascido em tepos de COVID-19: } \\
\text { Revisão Integrativa }\end{array}$ & $\begin{array}{c}\text { Identificar la evidencia científica sobre las buenas } \\
\text { prácticas en el cuidado del recién nacido, desde } \\
\text { la sala de partos hasta el hogar, en tiempos del } \\
\text { COVID-19 }\end{array}$ \\
\hline 2020 & $\begin{array}{l}\text { Alves Masca- } \\
\text { renhas VHA, et } \\
\text { al. (18) }\end{array}$ & $\begin{array}{l}\text { Care recommendations for parturient } \\
\text { and postpartum women and newborns } \\
\text { during the COVID-19 pandemic: A } \\
\text { scoping review }\end{array}$ & $\begin{array}{l}\text { Mapear los conocimientos actuales sobre las } \\
\text { recomendaciones para la atención del parto, el } \\
\text { alumbramiento y el recién nacido en el contexto del } \\
\text { nuevo coronavirus }\end{array}$ \\
\hline 2020 & $\begin{array}{l}\text { Lopes de Sousa } \\
\text { ÁF, et al. (19) }\end{array}$ & $\begin{array}{c}\text { Effects of COVID-19 Infection during } \\
\text { pregnancy and neonatal prognosis: } \\
\text { What is the evidence? }\end{array}$ & $\begin{array}{l}\text { Evaluar las pruebas actuales presentadas en la } \\
\text { literatura con respecto a los posibles riesgos de } \\
\text { infección por el virus COVID-19 en las mujeres } \\
\text { embarazadas y su consiguiente transmisión }\end{array}$ \\
\hline 2020 & $\begin{array}{l}\text { Procianoy RS, } \\
\text { et al. }(20)\end{array}$ & $\begin{array}{l}\text { Neonatal COVID-19: Little evidence } \\
\text { and the need for more information }\end{array}$ & $\begin{array}{c}\text { Debatir sobre los aspectos relacionados con los } \\
\text { cuidados neonatales (intensivos o no) en el contexto } \\
\text { de la pandemia de COVID-19 y la necesidad de } \\
\text { nuevos estudios }\end{array}$ \\
\hline 2020 & $\begin{array}{l}\text { Freitas BM, } \\
\text { et al.(21) }\end{array}$ & $\begin{array}{l}\text { Prevention and control measures for } \\
\text { neonatal COVID-19 infection: A } \\
\text { scoping review }\end{array}$ & $\begin{array}{l}\text { Identificar en la literatura las medidas de prevención } \\
\text { y control de la infección neonatal por COVID-19 }\end{array}$ \\
\hline 2020 & Moore TA (22) & $\begin{array}{l}\text { Overview of the care of mothers and } \\
\text { newborns with COVID-19; Joint } \\
\text { Position Statement }\end{array}$ & $\begin{array}{l}\text { Plantear las Recomendaciones de la Asociación } \\
\text { Nacional de Enfermeras Neonatales para guiar la } \\
\text { atención basada en la evidencia durante la pandemia }\end{array}$ \\
\hline 2020 & $\begin{array}{c}\text { Zeng } \\
\text { et al. }(23)\end{array}$ & $\begin{array}{l}\text { Antibodies in infants born to mothers } \\
\text { with COVID-19 pneumonia }\end{array}$ & $\begin{array}{l}\text { Evaluar la infección por COVID-19 en mujeres } \\
\text { embarazadas, mujeres puérperas y recién nacidos }\end{array}$ \\
\hline 2020 & $\begin{array}{l}\text { Dong } \\
\text { et al. }(24)\end{array}$ & $\begin{array}{l}\text { Possible vertical transmission of } \\
\text { SARS-CoV-2 from an infected mother } \\
\text { to her newborn }\end{array}$ & $\begin{array}{l}\text { Evaluar el SARS-CoV-2 en mujeres embarazadas, } \\
\text { puérperas y recién nacidos }\end{array}$ \\
\hline 2020 & $\begin{array}{c}\text { Zeng } \\
\text { et al. }(25)\end{array}$ & $\begin{array}{l}\text { Neonatal early-onset infection with } \\
\text { SARS-CoV-2 in } 33 \text { neonates born to } \\
\text { mothers with COVID-19 in Wuhan, } \\
\text { China }\end{array}$ & $\begin{array}{l}\text { Evaluar la infección por COVID-19 en los recién } \\
\text { nacidos }\end{array}$ \\
\hline
\end{tabular}




\section{RECOMENDACIONES PARA EL CUIDADO Y LA ATENCIÓN DE LOS RECIÉN NACIDOS PREMATUROS EN TIEMPOS DE LA PANDEMIA DE COVID-19: REVISIÓN INTEGRADA DE LA LITERATURA}

Tabla 2. Síntesis de los resultados de la revisión de literatura integrativa según: año, autor, título y objetivo. (cont.)

\begin{tabular}{|c|c|c|c|}
\hline Año & Autor(es) & Título & Objetivo(s) \\
\hline 2020 & $\begin{array}{c}\text { Zeng } \\
\text { et al. }(26)\end{array}$ & $\begin{array}{c}\text { First case of neonate infected with } \\
\text { novel coronavirus pneumonia in } \\
\text { China }\end{array}$ & $\begin{array}{c}\text { Describir el primer caso de un recién nacido } \\
\text { infectado con una nueva neumonía por coronavirus } \\
\text { en China }\end{array}$ \\
\hline 2020 & $\begin{array}{l}\text { Coronado } \\
\text { Munoz et al. } \\
\quad(27)\end{array}$ & $\begin{array}{l}\text { Late-onset neonatal sepsis in a patient } \\
\text { with COVID-19 }\end{array}$ & $\begin{array}{c}\text { Describir la sepsis neonatal de aparición tardía de } \\
\text { Covid-19 }\end{array}$ \\
\hline 2020 & $\begin{array}{l}\text { Vivanti } \\
\text { et al. }(28)\end{array}$ & $\begin{array}{l}\text { Transplacental transmission of SARS- } \\
\text { CoV-2 infection }\end{array}$ & $\begin{array}{l}\text { Demostrar por primera vez la transmisión } \\
\text { transplacentaria del SARS-CoV-2 en un recién } \\
\text { nacido de una madre infectada en el último trimestre }\end{array}$ \\
\hline 2020 & $\begin{array}{l}\text { Li F, } \\
\text { et al. }(29)\end{array}$ & $\begin{array}{c}\text { Proposal for prevention and control of } \\
\text { the } 2019 \text { novel coronavirus disease in } \\
\text { newborn infants }\end{array}$ & $\begin{array}{c}\text { Describir una propuesta para la prevención y el } \\
\text { control de la nueva enfermedad coronaviral de } 2019 \\
\text { en los recién nacidos }\end{array}$ \\
\hline 2020 & $\begin{array}{l}\text { de Souza J, } \\
\text { et al. }(30)\end{array}$ & $\begin{array}{l}\text { Family home care for newborns: an } \\
\text { ethnographic case study }\end{array}$ & $\begin{array}{l}\text { Analizar la dinámica del cuidado familiar con el } \\
\text { recién nacido en casa, en el municipio de Piraí-RJ }\end{array}$ \\
\hline
\end{tabular}

En la primera búsqueda en MEDLINE usando los términos: COVID-19, Cuidados de enfermería AND Recién nacidos prematuros, se obtuvieron 22 artículos, 16 de los cuales fueron seleccionados para leer el título y el resumen. Tras una lectura crítica y exhaustiva de los artículos seleccionados, se incluyeron 2 , ya que tratan sobre la enfermería neonatal y las infecciones por coronavirus. Posteriormente, se repitió el proceso en SCIELO utilizando los descriptores: COVID-19 AND Enfermería neonatal, dio lugar a 2 artículos, que fueron seleccionados para leer el título y el resumen, que después de la lectura crítica y detallada se incluyó 1 artículo, ya que aborda el tema de la atención de enfermería al recién nacido en un escenario pandémico por COVID-19, el otro artículo ya había sido incluido en la investigación. En PUBMED, utilizando los descriptores señalados anteriormente, se encontraron 11 artículos, que fueron seleccionados para leer el título y el resumen, después de lo cual, se incluyeron los 10 de ellos. Finalmente, se realizó la investigación en LILACS con los descriptores COVID-19 AND Nursing, lo que dio como resultado 2 artículos que fueron seleccionados para leer el título y el resumen, y de estos se incluyó 1 artículo y el otro ya había sido seleccionado (tabla 1) (17-30).

\section{RESULTADOS}

De los 31 estudios que se consideraron inicialmente aptos para la lectura de los títulos y resúmenes, solo 14 estudios presentaron datos sobre la atención a los recién nacidos en el escenario de la pandemia de COVID-19. Así pues, estos artículos se seleccionaron para su lectura completa y abordaje del tema, destacando los aspectos de la atención de enfermería al recién nacido en situación de riesgo durante la pandemia de la COVID-19 y, por tanto, constituyeron la muestra del estudio. Finalmente, en esta RIL se incluyeron seis artículos que se consideraron pertinentes para el tema propuesto (tabla 1).

Los estudios seleccionados se publicaron en revistas nacionales e internacionales, en el año 2020. De los artículos incluidos en el estudio, el 28,6\% $(n=4)$ se publicaron en revistas de enfermería $(17,18,21,30)$, 
el $21,4 \%(n=3)$ en revistas de pediatría y neonatología $(22,20,29)$, el $14,3 \%(n=2)$ en revistas de salud pública $(19,26)$ y el $35,75 \%(\mathrm{n}=5)$ en revistas de ciencias biomédicas $(23-25,27,28)$. En todos los artículos analizados, la mayoría de los autores tienen un doctorado $(\mathrm{n}=70)$ y en dos artículos había maestría en Ciencias de la Salud $(18,30)$. Según la información obtenida sobre los autores, alrededor del 37,8 \% $(\mathrm{n}=28)$ son enfermeras y el $62,2 \%(n=46)$ son médicos.

En cuanto al método utilizado por los artículos analizados, alrededor del 35,7 \% utilizó un estudio de caso $(n=5)$, el 21,4 \% $(n=3)$ utilizó la revisión de la literatura y el resto utilizó tanto la revisión del alcance para la síntesis de la evidencia como la elaboración de guías de práctica clínica y estudios retrospectivos, de cohorte y clínicos.

\section{DISCUSIÓN}

El propósito de la RIL es incluir el análisis de las investigaciones pertinentes para mejorar la práctica clínica, permitiendo el análisis de los conocimientos sobre un tema determinado, además de señalar la necesidad de nuevos estudios (16). La RIL es capaz de reunir y sintetizar estudios publicados y permitir conclusiones generales sobre un tema.

La realización de estudios en todas las regiones del país es de suma importancia, ya que el Brasil tiene importantes diferencias socioeconómicas entre sus regiones y es necesario que esos estudios puedan caracterizar el problema con las características específicas de sus respectivas regiones. La publicación de artículos en las revistas que se ocupan de estas áreas demuestra la preocupación por el debate de las pruebas actuales frente a la situación de emergencia de la salud pública, el enfoque en el desempeño de los profesionales que trabajan en primera línea y las repercusiones de esta pandemia en los grupos más vulnerables.
En todos los artículos analizados, la mayoría de los autores tienen doctorado o maestría en Ciencias de la Salud. Esta evidencia demuestra la correspondencia con el tema investigado, ya que el interés motivador de la investigación implica el cuidado proporcionado por la enfermería. Estos hallazgos también muestran el predominio de autores con altas calificaciones académicas en las producciones científicas más actuales. El uso de estos métodos demuestra la preocupación de los autores por publicar la información más actualizada disponible basada en los datos científicos generados en el escenario de la pandemia, en la que se insertan constantemente nuevos datos e información. La elevada tasa de estudios de revisión también refleja la urgencia de poner a disposición los datos y la información, ya que los ensayos clínicos tardan más en prepararse y publicarse, y los ensayos clínicos que ya han sido publicados tienen una muestra reducida.

En el análisis de los estudios, a fin de responder al problema de investigación de las recomendaciones en la atención del $\mathrm{RN}$, especialmente porque se trata de una población con inmadurez inmunológica y considerada en riesgo, han surgido datos sobre la atención sanitaria y la enfermería y la prevención de la aparición de la COVID-19, preparando a los servicios de salud para la detección de la enfermedad y la gestión temprana de los casos para controlar la pandemia y reducir el efecto ambiental de esta infección.

Entre las recomendaciones encontradas en los estudios analizados, se citó la importancia de la atención basada en la evidencia para orientar y realizar la práctica clínica, especialmente en estos tiempos sin precedentes, como base para la toma de decisiones y la educación continua de los profesionales $(20,22,27)$.

Los datos obtenidos en el estudio se clasificaron en fases para una mejor descripción de la información, que fueron: preparación para el momento del parto; 


\section{RECOMENDACIONES PARA EL CUIDADO Y LA ATENCIÓN DE LOS RECIÉN NACIDOS PREMATUROS EN TIEMPOS DE LA PANDEMIA DE COVID-19: REVISIÓN INTEGRADA DE LA LITERATURA}

medidas inmediatas después del parto; lactancia materna; y cuidado del $\mathrm{RN}$ de riesgo.

Las medidas relativas al momento del parto sugieren la evaluación conjunta de las condiciones maternas y fetales para elegir el mejor momento, vía de parto y lugar para el parto de la embarazada con sospecha o confirmación de COVID-19, dando prioridad al uso de una sala de presión negativa $(17,18,21)$ siempre que sea posible. Además, también se recomienda que las embarazadas con sospecha de infección utilicen una mascarilla quirúrgica como medida de precaución para evitar la transmisión del virus durante el parto (21). Los profesionales deben estar en el número mínimo necesario para el procedimiento y tener una protección estricta contra la infección, usando equipo de protección personal (PPE) adecuado durante todo el proceso del parto y en todos los procedimientos que puedan producir aerosoles (cuadro 1), como la intubación endotraqueal, la ventilación no invasiva, entre otros, y deben incluir el uso de gorros desechables y ropa protectora, cubiertas de zapatos, máscaras N95, gafas, guantes dobles e higiene de las manos $(17,18,20,21,31)$.

\section{Cuadro 1. Preparación para el momento del parto}

Preparación para el momento del parto

- Evaluación conjunta de las condiciones maternas y fetales para elegir el mejor momento, ruta de parto y lugar para el parto

- Priorizar el uso de una sala de presión negativa siempre que sea posible

- Uso de una mascarilla quirúrgica por parte de la embarazada como medida de precaución para evitar la transmisión del virus durante el parto

- Número mínimo posible de profesionales para el procedimiento y utilizar rigurosamente el equipo de protección personal (EPP) adecuado durante todo el proceso de parto y en todos los procedimientos que puedan producir aerosoles

Fuente: Adaptado do Ministério da Saúde. Secretaria de Atenção Primária à Saúde. Departamento de Ações Programáticas e Estratégicas (31).
Después del parto, a los recién nacidos de madres con sospecha o confirmación de la COVID-19 se les debe pinzar el cordón umbilical lo antes posible y ser limpiados y secados inmediatamente después del parto, aislados y examinados tempranamente para detectar la enfermedad (19,21). El contacto piel a piel también se desaconseja para un mejor control de la COVID-19 $(17,18,31)$, como se muestra en el cuadro 2.

Cuadro 2. Medidas inmediatas después del parto

Medidas inmediatas después del nacimiento

- Evite el pinzamiento tardío del cordón umbilical

- Se recomienda evitar el contacto piel a piel para reducir las fuentes potenciales de contaminación en el periodo inmediatamente posterior al parto

- El baño y el secado deben hacerse inmediatamente después del nacimiento para evitar la exposición y la hipotermia neonatal

Fuente: Adaptado do Ministério da Saúde. Secretaria de Atenção Primária à Saúde. Departamento de Ações Programáticas e Estratégicas (31).

Luego, después de la atención inicial del RN, se recomienda la separación temporal de la madre durante 14 días para minimizar el riesgo de transmisión viral. Si no es posible, debido a las limitaciones de la instalación o al deseo de la madre, mantenerse aproximadamente de 1 a 2 metros de distancia de la madre $(17,18)$. Si la puérpera con COVID-19 cuida al bebé, debe usar una mascarilla facial y practicar la higiene de las manos antes de cada comida u otro contacto cercano con el recién nacido (21).

En cuanto a la lactancia materna, recomiendan que se mantenga la práctica, siempre que la madre y el recién nacido estén en buenas condiciones clínicas y que se tomen todas las medidas preventivas durante el acto (las madres deben ponerse una mascarilla quirúrgica, higienizarse las manos y los utensilios de lactancia antes y después de cada toma) $(17,18)$. Algunos estudios han citado la posibilidad de extraer la leche materna, con la adopción de medidas como lavarse las 
manos antes de tocar el sacaleche o el recipiente de almacenamiento, limpiar adecuadamente el sacaleche y los utensilios utilizados después de cada uso y, si es posible, ofrecer la leche ordeñada al bebé $(21,31)$ según el cuadro 3.

Cuadro 3. Recomendaciones sobre lactancia materna

Lactancia materna

- Se recomienda mantener la práctica, siempre que se tomen todas las medidas preventivas durante el acto

- Las madres deben ponerse una mascarilla quirúrgica, desinfectarse las manos y los utensilios de lactancia antes y después de cada toma

- La extracción de la leche materna se puede hacer con la adopción de estrictas medidas de higiene $\mathrm{y}$, si es posible, otra persona debe ofrecer la leche al bebé

Fuente: Adaptado do Ministério da Saúde. Secretaria de Atenção Primária à Saúde. Departamento de Ações Programáticas e Estratégicas (31).

Los $\mathrm{RN}$ muestran signos y síntomas similares a los de los adultos infectados, como disnea, fiebre, taquicardia, vómitos y trombocitopenia. Por tanto, deben ser trasladados a aislamiento neonatal inmediatamente después de ser evaluados por el neonatólogo y se les debe controlar cuidadosamente la frecuencia respiratoria, la temperatura corporal, la frecuencia cardíaca y los signos y síntomas gastrointestinales. Lo ideal sería que el $\mathrm{RN}$ permaneciera en un pabellón aislado y que su estado clínico fuera constantemente monitoreado. Se recomienda la intervención temprana tan pronto como se observen patrones anormales y estos deben ser el principal foco de atención después del nacimiento (18). Los RN con confirmación de la COVID-19 necesitan la aplicación inmediata de medidas de precaución, con aislamiento de contacto, gotas y aerosoles garantizado, y se recomienda que se mantengan en una Unidad de Cuidados Intensivos Neonatales (UCIN) con un periodo de cuarentena de al menos 14 días, con la separación temporal de su madre durante 14 días para minimizar el riesgo de transmisión viral (21).
En los casos de sospecha descartada o después de cumplir el período de cuarentena, es conveniente mantener la sala del RN a niveles apropiados de temperatura y humedad, además de ventilada y preferiblemente con las ventanas abiertas. También se recomienda reducir al mínimo las visitas y el contacto físico. Las manos y la cara deben limpiarse frecuentemente al hacer contacto y el suelo y los muebles deben limpiarse y desinfectarse con alcohol al $70 \%$ o compuestos de cloro disueltos y los chupetes y botellas de material resistente al calor deben desinfectarse a altas temperaturas $(21,31)$ (cuadro 4$)$.

Cuadro 4. Cuidado del recién nacido en riesgo

Cuidado del recién nacido en riesgo

- $\quad$ El RN necesita permanecer en un pabellón aislado y tener su condición clínica constantemente monitoreada

- Controlar rigurosamente frecuencia respiratoria, temperatura corporal, frecuencia cardíaca, signos y síntomas gastrointestinales

- Intervención temprana tan pronto como se observen patrones anormales y estos deben ser el principal foco de atención después del nacimiento

- En los casos sospechosos o confirmados de COVID-19, mantenga al bebé en la Unidad de Cuidados Intensivos Neonatales (UCIN) y complete un periodo de cuarentena de al menos 14 días con la separación temporal del bebé y su madre durante 14 días

- En los casos de sospecha descartada o después de cumplir el periodo de cuarentena, es conveniente mantener la sala del $\mathrm{RN}$ a niveles apropiados de temperatura y humedad, ventilada y preferiblemente con las ventanas abiertas

- Reducir al mínimo las visitas y el contacto físico, limpiar las manos y la cara con frecuencia y desinfectar el suelo y los muebles con alcohol al $70 \%$ o compuestos de cloro disueltos

- Los chupetes y biberones de material resistente al calor deben ser desinfectados a altas temperaturas

Fuente: Adaptado do Ministério da Saúde. Secretaria de Atenção Primária à Saúde. Departamento de Ações Programáticas e Estratégicas (31). 


\section{RECOMENDACIONES PARA EL CUIDADO Y LA ATENCIÓN DE LOS RECIÉN NACIDOS PREMATUROS EN TIEMPOS DE LA PANDEMIA DE COVID-19: REVISIÓN INTEGRADA DE LA LITERATURA}

A juzgar por la ausencia de datos coherentes y la constante producción de nueva información sobre la infección por coronavirus y sus repercusiones, la prematuridad no está directamente relacionada con la infección materna por la COVID-19, así como la infección no representa una gravedad en caso de prematuridad. Por tanto, las medidas de precaución y todas las recomendaciones descritas anteriormente equivalen a la gestión del $\mathrm{RN}$ en el contexto de la pandemia.

\section{CONSIDERACIONES FINALES}

Desde el comienzo de la pandemia de COVID-19 en China, los ojos de la comunidad científica han estado puestos en el control, el diagnóstico y la cura de esta infección. Sin embargo, otras áreas de la salud siguen exigiendo atención, incluso durante esta pandemia, como el embarazo y el parto, que siguen cumpliendo su ciclo y potenciando las dudas respecto a los cuidados de los RN, desde la sala de partos hasta el alta hospitalaria.

Con relación a las enfermeras, todavía hay poca información sobre las manifestaciones clínicas de la COVID-19. La mayoría de los estudios analizados muestran que estas manifestaciones de la enfermedad son menos graves en los niños que en los adultos. Porotro lado, los RN necesitan una atención especial, ya que su sistema inmunológico es todavía inmaduro, lo que los hace más susceptibles a la infección por el coronavirus. En lo que respecta a la transmisión del coronavirus, su tratamiento y otras condiciones relacionadas con los recién nacidos, todavía hay mucho por conocer $\mathrm{y}$, por tanto, se están realizando investigaciones en todo el mundo. Se entiende que en la mayoría de los casos la transmisión puede producirse por contacto directo con las gotas respiratorias de personas infectadas o por contacto indirecto con superficies del medio ambiente u objetos contaminados.
Con el fin de evitar la propagación del virus, los profesionales de la salud deben adoptar medidas de precaución estrictas, además de ofrecer orientación sobre esas medidas, entre ellas lavar las manos de la madre antes de tocar al bebé, utilizar una mascarilla durante la lactancia y permanecer aislados en viviendas conjuntas. Debido a la ausencia de pruebas de transmisión vertical, así como de transmisión a través de la leche materna, la mayoría de las organizaciones científicas recomiendan no separar a las madres y los lactantes, con el fin de promover la lactancia materna y el vínculo neonatal, con la excepción de los casos con síntomas maternos graves, y en esos casos se sugieren medidas de barrera física, así como la administración de leche materna ordeñada.

Por último, se entiende la necesidad de que las enfermeras y otros profesionales de la salud conozcan las medidas de prevención y control de la infección COVID-19 durante la atención, a fin de promover una atención actualizada y basada en pruebas. Solo con medidas rigurosas de prevención y control será posible reducir el impacto de la pandemia en la población neonatal.

\section{REFERENCIAS}

1. Esakandari H, Nabi-Afjadi M, Fakkari-Afjadi J, Farahmandian N, Miresmaeili SM, Bahreini E. A comprehensive review of COVID-19 characteristics. Biol Proced Online. 2020;22:19 doi: https://doi. org/10.1186/s12575-020-00128-2

2. Ministério da Saúde Brasil [Internet]. Brasilia: Coronavírus COVID-19. 2020 [consultado en agosto de 2020]. Disponible em: https://coronavirus.saude. gov.br

3. Ministério da Saúde Brasil [Internet]. Brasilia: Protocolo de Manejo Clínico para o Novo Coronavírus (2019-nCoV). 2020 [consultado en agosto de 2020] Disponible en: https://portalarquivos2.saude.gov. br/images/pdf/2020/fevereiro/11/protocolo-manejocoronavirus.pdf 
4. Werneck GL, Carvalho MS. A pandemia de COVID-19 no Brasil: crônica de uma crise sanitária anunciada. Cad. Saúde Pública. 2020; 36(5):e00068820 http:// dx.doi.org/10.1590/0102-311X00068820

5. Garcia LP. Uso de máscara facial para limitar a transmissão da COVID-19. Artigo de opinião. Epidemiol Serv Saúde. 2020; 29 (2): [4 páginas]. doi: https://doi.org/10.5123/S1679-49742020000200021

6. Zucco L, Levy N, Ketchandji D, Aziz M, Ramachandran SK. Considerações perioperatórias para o novo coronavírus 2019 (COVID- 19). Anesthesia Patient Safety Foundation (APSF) Boletin apsf [Internet]. 2020 [consultado en agosto de 2020]; 3(2):36-40. Disponible en: https://www.apsf.org/wp-content/ uploads/newsletters/2020/0302-pt-br/APSF0302-PTBR.pdf

7. Ribeiro-Furlan MC, Jurado SR, Uliana $\mathrm{CH}$, Pascoaloto ME, Nagata LA, Freitas AC. Gravidez e infecção por Coronavírus: desfechos maternos, fetais e neonatais - Revisão sistemática. Rev Cuid. 2020; 11(2): e1211. doi: http://dx.doi.org/10.15649/cuidarte.1211

8. Sociedade Brasileira de Pediatria. Departamento Científico de Neonatologia da SPSP [Internet]. Copacabana: Coronavírus e Recém-Nascido: o que se sabe até o momento? Versão 3. 2020 [consultado en agosto de 2020]. Disponible en: https://www. spsp.org.br/PDF/SPSP-DC\%20Neonatologia-Covidvers\%C3\%A3o3-25.09.2020.pdf

9. Dong Y, Mo X, Hu Y, Qi X, Jiang F, Jiang Z, et al. Epidemiological characteristics of 2143 pediatric patients with 2019 coronavirus disease in China. Pediatrics. 2020:e20200702. doi: 10.1542/peds.20200702

10. $\mathrm{Wu} \mathrm{Z}$, McGoogan JM. Characteristics of and Important Lessons From the Coronavirus Disease 2019 (COVID-19) Outbreak in China: Summary of a Report of 72314 Cases From the Chinese Center for Disease Control and Prevention. JAMA. 2020; 323(13):12391242. doi: 10.1001/jama.2020.2648.

11. Livingston E, Bucher K. Coronavirus Disease 2019 (COVID-19) in Italy. JAMA. 2020; 323(14):1335. doi: 10.1001/jama.2020.4344.

12. DeAlmeida MF, Guinsburg R, editoras. Recomendações para Assistência ao Recém-Nascido na sala de parto de mãe com COVID-19 suspeita confirmada [Internet]. Copacabana: Sociedade Brasileira de Pediatria; 2020 [consultado en agosto de 2020]. Disponible en: https:// www.sbp.com.br/fileadmin/user_upload/22422bNAlerta-Assist_RN_SalaParto_de_mae_com_ COVID-19.pdf
13. Santiago Rego MA, editora. Recomendações sobre os cuidados respiratórios do recém-nascido com COVID-19 SUSPEITA ou CONFIRMADA [Internet]. Copacabana: Sociedade Brasileira de Pediatria; 2020 [consultado en agosto de 2020]. Disponible en: https:// www.sbp.com.br/fileadmin/user_upload/22428b-DC_ RED_-_Recom_cuidados_Respir_RN_com_COVID. pdf

14. Santiago Rego MA, editora. Prevenção e Abordagem da Infecção por COVID-19 em mães e Recém-Nascidos, em Hospitais- Maternidades [Internet]. Copacabana: Sociedade Brasileira de Pediatria; 2020 [consultado en agosto de 2020]. Disponible en: https://www.sbp. com.br/fileadmin/user_upload/22412b-Nota_Alerta PrevenAbordagem_infeccao_COVID19_maes-RN_ em_HospMatern.pdf

15. Ministério da Saúde Brasil [Internet]. Brasilia: Secretaria de Atenção Primária à Saúde. Nota Técnica $\mathrm{N}^{\mathrm{0}}$ 6/2020-COCAM/CGCIVI/DAPES/SAPS/MS; 2020 [consultado en agosto de 2020] Disponible en: https://docs.bvsalud.org/biblioref/2020/04/1087595/ notatecnicaneonatal30mar2020covid-19.pdf

16. Tavares de Souza M, Silva, Dias da Silva M, de Carvalho R. Revisão integrativa: o que é e como fazer. Einstein (São Paulo), 8(pt1):102-106. https://doi.org/10.1590/ s1679-45082010rw1134

17. Garcia F, Torres AS, Lucchese I, da Silva LJ, da Silva LF, Silva MA. Boas práticas no cuidado ao recémnascido em tempos de COVID-19: revisão integrativa. Texto Contexto Enferm. 2020;29: e20200242. doi: https://doi.org/10.1590/1980-265X-TCE-2020-0242

18. Alves Mascarenhas VH, Caroci-Becker A, Pereira KC, Girardi N, Caroci A, Gonzalez ML. Care recommendations for parturient and postpartum women and newborns during the COVID-19 pandemic: a scoping review. Rev Latino-Am Enfermagem. 2020; 28:e3359. doi: http://dx.doi.org/10.1590/15188345.4596 .3359

19. Lopes de Sousa ÁF, Carvalho HEF, Oliveira LB, Schneider G, Camargo ELS, Watanabe E, et al. Effects of COVID-19 Infection during Pregnancy and Neonatal Prognosis: What Is the Evidence? Int J Environ Res Public Health. 2020; 17(11):4176. doi: 10.3390/ ijerph17114176.

20. Procianoy RS, Silveira RC, Manzoni P, Sant'Anna G. Neonatal COVID-19: little evidence and the need for more information. J Pediatr (Rio J). 2020; 96(3):269272. doi: 10.1016/j.jped.2020.04.002.

21. Freitas BHBM, Alves MDSM, Gaíva MAM. Prevention and control measures for neonatal COVID-19 infection: 


\section{RECOMENDACIONES PARA EL CUIDADO Y LA ATENCIÓN DE LOS RECIÉN NACIDOS PREMATUROS EN TIEMPOS DE LA PANDEMIA DE COVID-19: REVISIÓN INTEGRADA DE LA LITERATURA}

a scoping review. Rev Bras Enferm. 2020; 73 Suppl 2:e20200467. Portuguese, English. doi: 10.1590/00347167-2020-0467.

22. Moore TA. Overview of the Care of Mothers and Newborns With COVID-19; Joint Position Statement. Adv Neonatal Care. 2020; 20(4):268. doi: 10.1097/ ANC.0000000000000776.

23. Zeng H, Xu C, Fan J, Tang Y, Deng Q, Zhang W, et al. Antibodies in Infants Born to Mothers With COVID-19 Pneumonia. JAMA. 2020; 323(18):1848-1849. doi: 10.1001/jama.2020.4861.

24. Dong L, Tian J, He S, Zhu C, Wang J, Liu C, et al. Possible Vertical Transmission of SARS-CoV-2 From an Infected Mother to Her Newborn. JAMA. 2020; 323(18):1846-1848. doi: 10.1001/jama.2020.4621.

25. Zeng L, Xia S, Yuan W, Yan K, Xiao F, Shao J, et al. Neonatal Early-Onset Infection With SARS-CoV-2 in 33 Neonates Born to Mothers With COVID-19 in Wuhan, China. JAMA Pediatr. 2020; 174(7):722-725. doi: 10.1001/jamapediatrics.2020.0878.

26. Zeng LK, Tao XW, Yuan WH, Wang J, Liu X, Liu ZS. [First case of neonate infected with novel coronavirus pneumonia in China]. Zhonghua $\mathrm{Er} \mathrm{Ke} \mathrm{Za}$ Zhi. 2020; 58(0):E009. Chinese. doi: 10.3760/cma.j.is sn.0578-1310.2020.0009.

27. Coronado Munoz A, Nawaratne U, McMann D, Ellsworth M, Meliones J, Boukas K. Late-Onset Neonatal Sepsis in a Patient with Covid-19. N Engl J Med. 2020;382(19):e49. doi: 10.1056/NEJMc2010614.
28. Vivanti AJ, Vauloup-Fellous C, Prevot S, Zupan V, Suffee C, Do Cao J, et al. Transplacental transmission of SARS-CoV-2 infection. Nat Commun. 2020; 11(1):3572. doi: 10.1038/s41467-020-17436-6.

29. Li F, Feng ZC, Shi Y. Proposal for prevention and control of the 2019 novel coronavirus disease in newborn infants. Arch Dis Child Fetal Neonatal Ed. 2020; 105(6):683684. doi: 10.1136/archdischild-2020-318996.

30. de Souza J, Moreira M. Cuidado familial de recémnascidos no domicílio: um estudo de caso etnográfico. Texto Contexto - Enferm. 2011; 20: 223-231. doi: https://doi.org/10.1590/S0104-07072011000500028

31. Ministério da Saúde Brasil. Secretaria de Atenção Primária à Saúde. Departamento de Ações Programáticas e Estratégicas [Internet]. Brasilia: Manual de Recomendações para a Assistência à Gestante e Puérpera frente à Pandemia de Covid-19. 2020 [consultado en agosto de 2020] Disponible en: http://189.28.128.100/dab/docs/portaldab/documentos/ corona/manual_recomendacoes_gestantes_covid19. pdf 